\title{
Combination of Converging Collimators for High-Sensitivity Brain SPECT
}

\author{
Ruben Ter-Antonyan ${ }^{1}$, Ronald J. Jaszczak ${ }^{1,2}$, Kim L. Greer ${ }^{1}$, James E. Bowsher ${ }^{3}$, Scott D. Metzler ${ }^{4}$, and \\ R. Edward Coleman ${ }^{1}$
}

${ }^{1}$ Department of Radiology, Duke University Medical Center, Durham, North Carolina; ${ }^{2}$ Department of Biomedical Engineering, Duke University, Durham, North Carolina; ${ }^{3}$ Department of Radiation Oncology, Duke University Medical Center, Durham, North Carolina; and ${ }^{4}$ Department of Radiology, University of Pennsylvania, Philadelphia, Pennsylvania

The objective of this study, which is related to human brain SPECT, was to increase the sensitivity of a triple-camera SPECT system and reduce statistical noise in reconstructed images using a combination of converging collimators. The reason for combining collimators is to ensure both high sensitivity and sufficient sampling without trading off spatial resolution. Methods: A highsensitivity half-cone-beam (HCB) collimator, designed specifically for brain imaging, was combined with other collimators and compared with conventional parallel-beam and fanbeam circular orbit acquisitions. For comparison, previously studied HCB collimation with a circle-and-helix data acquisition trajectory was also included in this study. Simulations of the Hoffman 3dimensional brain phantom were performed to calculate the efficiencies of collimators and their combinations and to quantitatively evaluate reconstruction bias, statistical noise, and signalto-noise ratios in the reconstructed images. Experimental brain phantom data were also acquired and compared for different acquisition types. Finally, a patient brain scan was obtained with a combination of HCB and fanbeam collimators and compared with a triple-fanbeam circular orbit acquisition. Results: A combination of 2 HCB collimators and 1 fanbeam collimator, compared with a triple-fanbeam collimator, can increase the photon detection efficiency by $27 \%$ and by more than a factor of 2, compared with triple-parallel-hole collimation, with equal spatial resolution measured on the axis of rotation. Quantitative analysis of reconstruction bias and visual analysis of the images showed no signs of sampling artifacts. Reconstructed images in the simulations, experimental brain phantom, and patient brain scans showed improved quality with this collimator combination due to increased sensitivity and reduced noise. Lesion visibility was also improved, as confirmed by signal-to-noise ratios. Alternatively, triple-HCB circle-and-helix acquisition has also shown competitive results, with a slight disadvantage in axial sampling and implementation procedure. Conclusion: Combined HCB and fanbeam collimation is a promising approach for high-sensitivity brain SPECT.

Key Words: brain SPECT; combined collimation; half-conebeam; triple-camera; circle-and-helix

J Nucl Med 2009; 50:1548-1556

DOI: 10.2967/jnumed.109.062653

Received Jan. 27, 2009; revision accepted May 22, 2009.

For correspondence or reprints contact: Ruben Ter-Antonyan, Department of Radiation Oncology, University of Virginia Health System 800375, Charlottesville, VA 22908.

E-mail: rt3ak@hscmail.mcc.virginia.edu

COPYRIGHT @ 2009 by the Society of Nuclear Medicine, Inc.
$I_{1}$ n SPECT, collimator sensitivity and spatial resolution, along with sufficient sampling of the object, define the reconstructed image quality. High sensitivity and resolution are essential for reducing statistical noise and improving the level of detail in the images. Converging collimators such as fanbeam and cone-beam offer increased sensitivity, compared with parallel-beam (PB) collimators that are often used in clinical patient scans. Cone-beam collimators are more sensitive than are fanbeam collimators because they are converging both axially and transaxially. For improved resolution in brain imaging, the $\gamma$-camera must be located close to the brain and not interfere with the patient's shoulders. For many SPECT systems, this means that the use of a cone-beam collimator may result in the truncation of projection data from the caudal portion of the brain. Alternatively, a half-cone-beam (HCB) collimator with the focal point shifted toward the base of the brain offers sensitivity and resolution similar to a cone-beam collimator but avoids truncation (1), provided the field of view (FOV) of the camera is axially large enough to fit the magnified projection image.

A conventional circular orbit (CO) data acquisition with an HCB-collimated $\gamma$-camera may result in axial distortions in the reconstructed images due to insufficient axial sampling of the brain, even when the entire brain is inside the FOV of the camera. To alleviate the axial artifacts, helical HCB data acquisition has been previously investigated with a triple-camera SPECT system (2,3). Helical SPECT scans have been implemented by translating the patient axially as the gantry rotates in a CO. The combined rotational motion of the gantry and the patient axial translation result in a helical trajectory of the collimator focal point around the brain. Similar to helical SPECT proposed for long-object cone-beam imaging (4-6), pinhole imaging (7), and CT scans $(8-10)$, helical triple-HCB scans demonstrated markedly improved axial sampling and showed no signs of axial artifacts in simulation and experiments $(2,3)$.

Alternatively, multicamera SPECT systems may combine different types of collimators to improve both sensitivity and axial sampling (11-17). For the triple-camera 
system described here, a combination of HCB and fanbeam collimators may offer artifact-free reconstructed images with reduced noise. In this article, various collimator combinations are studied and compared with triple-HCB circle-andhelix $(\mathrm{CH})$, triple-fanbeam, and triple-PB $\mathrm{CO}$ acquisitions to determine which combinations of the collimators may offer the largest potential for improved brain SPECT. Similar studies were performed recently by our group using a Defrise disk phantom (Data Spectrum Corp.) for simulation and experimental imaging and showed promising results for a combination of HCB and fanbeam collimators (18).

\section{MATERIALS AND METHODS}

\section{Collimator Combinations and Data Acquisition Trajectories}

The following collimator combinations and data acquisition trajectories for triple-camera brain SPECT were studied: HCB + $\mathrm{HCB}+$ fanbeam, $\mathrm{CO} ; \mathrm{HCB}+$ fanbeam + fanbeam, $\mathrm{CO} ; \mathrm{HCB}+$ full-cone-beam $(\mathrm{FCB})+$ fanbeam, $\mathrm{CO}$; triple-HCB, $\mathrm{CH}$ trajectory; triple-fanbeam, $\mathrm{CO}$; and triple- $\mathrm{PB}, \mathrm{CO}$.

These combinations were compared for photon detection efficiency and reconstructed image quality. Parallel-hole collimators were studied as a reference. We assumed that the $\mathrm{HCB}+\mathrm{PB}$ combination was less efficient than the $\mathrm{HCB}+$ fanbeam combination and therefore do not consider it here. A CO implies that each of the 3 SPECT cameras, equally separated by $120^{\circ}$, rotates for $360^{\circ}$. CO sampling was $1^{\circ}$ during simulation and experimental phantom studies and $2^{\circ}$ during a patient brain scan. A $\mathrm{CH}$ trajectory implies a $300^{\circ}$ circle followed by a $60^{\circ}$ helix. Thus, acquisition time for the $\mathrm{CO}$ is 5 times longer than that for the helix, which keeps the phantom longer in the FOV and increases the photon detection efficiency. We studied whether a short-term helical trajectory with HCB collimation proposed here was sufficient for improved axial sampling. The total number of views and time per view during all data acquisitions were constant.

\section{Disk Brain Phantom Monte Carlo Simulation: Collimator Parameters and Geometric Efficiency}

Collimator efficiency, defined as a ratio between the number of photons detected by the $\gamma$-camera and the number emitted by the source, was calculated using Monte Carlo simulation. A disk phantom (9 disks with different radii, each $1.28 \mathrm{~cm}$ thick) superficially resembling the Hoffman 3-dimensional (3D) brain phantom (model BR/3D/P; Data Spectrum Corp.) in shape and size was used in the simulations. The disk radii were $6.8,8.1,8.4,8.3,7.9,7.4$, 6.7, 5.7, and $2.9 \mathrm{~cm}$, listed in a caudal-to-cranial order. The phantom was centered on the axis of rotation (AOR), located $13 \mathrm{~cm}$ from the collimator surface. This distance is also called the radius of rotation (ROR) of the camera. The phantom disks were perpendicular to the AOR and symmetric transverse to it. The activity in the disks was assumed uniform. Similar to the physical Hoffman brain phantom, the simulated disks were located inside a cylinder (diameter, $18 \mathrm{~cm}$; height, $12.24 \mathrm{~cm}$ ). The disk brain phantom and the HCB collimator are illustrated in Figure 1. The collimator focal point was located at $50 \mathrm{~cm}$ from the collimator surface (shaded area, Fig. 1), defined here as the surface that is closer to the AOR. The HCB focal point was shifted axially by $11.5 \mathrm{~cm}$ toward the base of the phantom as measured from the center of the collimator. The focal lengths of the converging collimators were equal. All collimator FOVs were $40 \times 23 \mathrm{~cm}$,

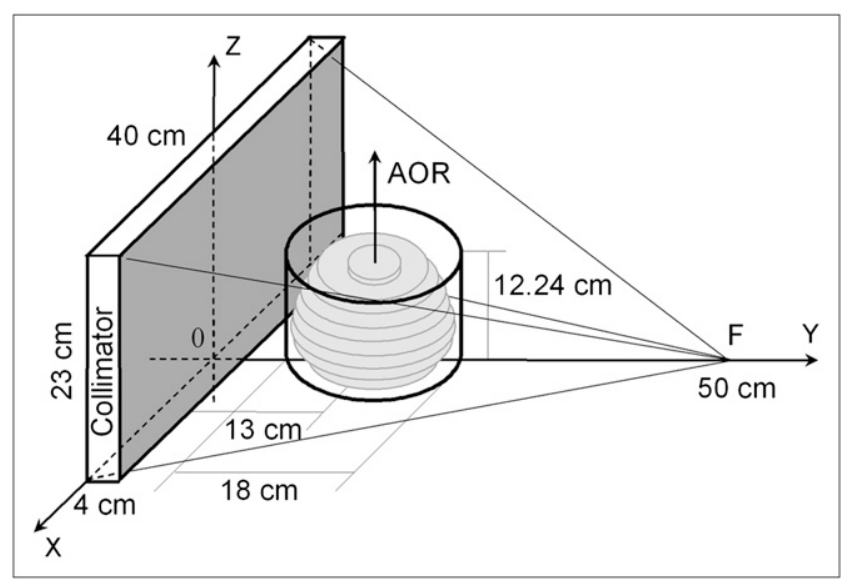

FIGURE 1. 3D sketch of HCB collimator and disk brain phantom used in Monte Carlo simulation. Phantom and collimator dimensions are shown. Phantom is centered on AOR, $13 \mathrm{~cm}$ from collimator surface (shaded).

corresponding to the camera FOV. The collimators were $4 \mathrm{~cm}$ thick. Converging collimators were simulated with square holes (size, $0.145 \mathrm{~cm}$ ) and $0.022-\mathrm{cm}$ septal thickness. The hole-size parameter of the simulated parallel-hole collimator was adjusted to $0.132 \mathrm{~cm}$ so that the spatial resolutions of all collimators calculated on the AOR were similar. Collimator spatial resolution was modeled to include both geometric and detector intrinsic (full width at half maximum [FWHM], $0.35 \mathrm{~cm}$ ) resolutions.

Collimator geometric efficiencies vary depending on the location of the source emitting $140-\mathrm{keV}$ photons. This means that photons from a certain region of the brain may have a higher or lower probability of being detected than photons from other regions. The issue is further complicated by photon attenuation and scatter inside the brain. To understand how different parts of the brain are affected by the collimator geometry, photon detection efficiencies were studied for each of the 9 phantom disks. Photon attenuation and scatter in the disks and the cylinder were modeled using the attenuation coefficient for water $\left(\mu=0.15 \mathrm{~cm}^{-1}\right)$. The energies of the scattered photons were computed from the scattering angle, which was simulated using the Klein-Nishina formula for differential cross-section with respect to the solid angle of scattering. Photons that passed through the collimator were detected if they had energies between 126 and $140 \mathrm{keV}$. Statistical uncertainty in collimator efficiency values was less than $2 \%$. Photon septal penetration and photopeak detection efficiency were not simulated, because they are mostly independent of the collimator types studied here.

\section{Hoffman Brain Phantom SPECT Simulation}

Along with the Monte Carlo simulation of a simplified uniform disk brain phantom to compute collimator efficiency for different disks in the phantom, we used the bitmaps of the Hoffman 3D brain phantom for projection and reconstruction in our SPECT simulation. The phantom models 19 different transaxial brain slices. To evaluate lesion visibility with the collimator configurations studied here, we placed (modeled) 3 hot and 3 cold spheres (1.1-cm diameter) in the bottom, middle, and top parts of the brain, as shown in Figure 2 bitmaps. Uptake ratios were 30\% higher for the hot spheres and $70 \%$ lower for the cold spheres than 


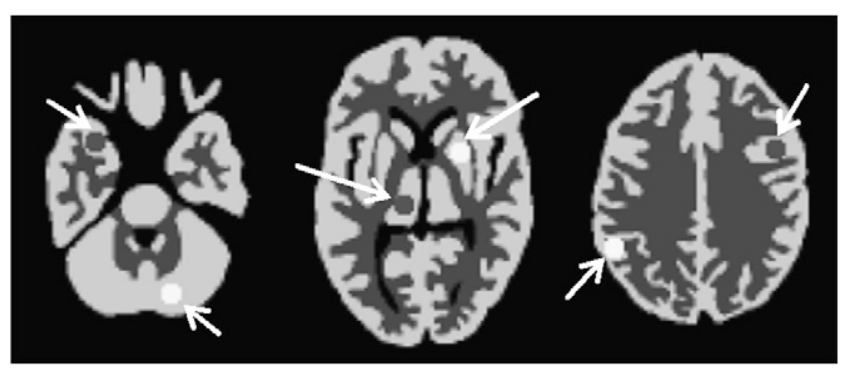

FIGURE 2. Hoffman 3D brain phantom slices (bitmaps) corresponding to slice numbers 1,6 , and 11 (of 19) in caudal-to-cranial order. Three hot spheres (30\% hotter than gray matter) were inserted into cerebellum (slice 1), basal ganglia (slice 6), and right parietal lobe (slice 11) of brain phantom. Three cold spheres (70\% colder than gray matter) were inserted into right temporal lobe (slice 1), thalamus (slice 6), and left frontal lobe (slice 11). Arrows in figure point to lesions.

they were for gray matter. The gray matter-white matter-ventricle uptake ratio in the Hoffman brain phantom used here is 4:1:0.

Projection data were simulated on a $256 \times 128$ grid using a raydriven forward-projection algorithm. The simulated Hoffman brain phantom was reconstructed iteratively, using an ordered-subsets expectation maximization (OSEM) algorithm (19) with 24 subsets and 5 iterations for converging collimator data. The OSEM convergence rate was slightly lower for parallel-hole data and required 7 iterations to achieve bias values similar to fanbeam. Collimator resolution and sensitivity were not modeled in the ray-driven forward-projection and OSEM reconstruction algorithms. The detector intrinsic resolution of $0.35 \mathrm{~cm}$ in FWHM, modeled as gaussian, was used only for noisy projection data. Attenuation was modeled using a uniform map based on the phantom dimensions. The attenuation coefficient used was $\mu=0.15 \mathrm{~cm}^{-1}$. Scatter and septal penetration effects were not modeled in simulations. Noise-free data were used to calculate reconstruction bias with respect to the Hoffman brain phantom bitmaps. Bias was calculated for each brain slice using

$$
\delta=\sum_{i=1}^{N}\left|g_{i}^{\text {noise-free }}-g_{i}^{\text {bitmap }}\right|,
$$

where $\mathrm{N}$ is the number of all pixels in a brain slice, and $\mathrm{g}_{\mathrm{i}}^{\text {noise-free }}$ and $g_{i}^{\text {bitmap }}$ are ith pixel activities in noise-free reconstructed and bitmap images. Slice-by-slice bias calculation demonstrates whether there are parts of the phantom that are more distorted than others.
For instance, an $\mathrm{HCB} \mathrm{CO}$ scan is expected to result in larger distortions in the cranial portions of the brain because of insufficient axial sampling, which will in turn be revealed quantitatively by larger bias values for cranial brain slices. The detector intrinsic resolution was not considered during bias calculation to avoid possible nonsampling artifacts (e.g., blur) affecting bias values.

Noisy projection data were obtained by generating an ensemble of 32 Poisson noise realizations based on noise-free bitmap projection data with a mean value of $2.7 \times 10^{7}$ counts per fanbeam-collimated camera. The numbers of counts for other simulated collimators were scaled according to their efficiencies. The level of noise in reconstructed images for a single noise realization was evaluated slice by slice using

$$
\sigma=\sqrt{\frac{1}{N} \sum_{i=1}^{N}\left(g_{i}^{\text {noisy }}-g_{i}^{\text {noise-free }}\right)^{2}},
$$

for all of the pixels $(\mathrm{N})$ in a brain slice. This value was then averaged over $\mathrm{M}=32$ noise realizations. Quantitatively speaking, the purpose of combining converging collimators in this study is to improve brain SPECT by decreasing both $\delta$ and $\sigma$ without altering spatial resolution.

Noise reduction should positively affect the brain lesion visualization, which can be quantitatively evaluated using signal-tonoise ratios (SNRs) for the hot and cold spheres (signals) inside the simulated Hoffman brain phantom:

$$
\text { SNR }=\frac{\sum_{j=1}^{M}\left(g_{j}^{\text {signal }}-g_{j}^{b k g}\right)}{\sum_{j=1}^{M} \sigma_{j}^{b k g}} .
$$

Here, $\mathrm{g}_{\mathrm{i}}^{\text {signal }}$ and $\mathrm{g}_{\mathrm{i}}^{\mathrm{bkg}}$ are normalized activities in the signal and the background area around it, and $\sigma_{j}^{\text {bkg }}$ is the normalized background noise calculated using Equation 2 for $\mathrm{N}$ background pixels.

\section{Experimental Imaging of Brain Phantom}

Experimental data were acquired using a triple-camera SPECT system (Triad XLT; Trionix Research Laboratories). Collimator specifications are shown in Table 1 . The fanbeam collimator used in this study had slightly better resolution because of a smaller hole size. The resulting loss of sensitivity was partially compensated for by a shorter fanbeam focal length. The focal point of the HCB collimator is unintentionally shifted $6 \mathrm{~mm}$ beyond the axial

\begin{tabular}{|c|c|c|c|c|c|c|}
\hline $\begin{array}{l}\text { Collimator } \\
\text { type }\end{array}$ & $\begin{array}{l}\text { Focal } \\
\text { length* } \\
(\mathrm{cm})\end{array}$ & $\begin{array}{l}\text { Hex hole } \\
\text { size } \\
\text { (cm) }\end{array}$ & $\begin{array}{l}\text { Septal } \\
\text { thickness } \\
\text { (cm) }\end{array}$ & $\begin{array}{c}\text { Hole } \\
\text { length } \\
(\mathrm{cm})\end{array}$ & $\begin{array}{l}\text { Resolution } \\
\text { FWHM }^{\dagger} \\
(\mathrm{cm})\end{array}$ & $\begin{array}{c}\text { Point source } \\
\text { sensitivity }{ }^{\dagger}(\text { counts } / \mathrm{s}) / \mathrm{kBq}\end{array}$ \\
\hline PB & Infinity & 0.138 & 0.016 & 4.54 & 0.65 & 164.28 \\
\hline FB & 39 & 0.122 & 0.015 & 4.13 & 0.56 & 255.30 \\
\hline $\mathrm{HCB}$ & 50 & 0.145 & 0.022 & 4.00 & 0.66 & 447.70 \\
\hline
\end{tabular}
extent of the $40 \times 23 \mathrm{~cm}$ FOV of the camera, which is considered during image reconstruction. The Hoffman 3D brain phantom 
described earlier was used in the experimental study. Each of the 19 Hoffman brain phantom slices was approximately $6.4 \mathrm{~mm}$ thick and consisted of 5 polycarbonate sublayers. The brain slices were placed in a polymethyl methacrylate cylinder (height, 17.5 $\mathrm{cm}$; diameter, $20.8 \mathrm{~cm}$ ). The phantom was filled with $1.4 \mathrm{~L}$ of water solution containing ${ }^{99 \mathrm{~m}} \mathrm{Tc}$ radiotracer $\left(1.9 \times 10^{5} \mathrm{~Bq} / \mathrm{cm}^{3}\right)$. There was no radioactivity in the cylinder outside the brain. Similar to simulation studies, 3 cold lesions were placed inside the physical phantom. The spheroid-shaped (grapelike) lesions had a volume of $3 \mathrm{~mL}$ and were filled with water with no activity. They were inserted into the cerebellum, thalamus, and frontal lobe of the brain phantom.

The 3 SPECT detectors were equally spaced by $120^{\circ}$ and acquired data simultaneously. The detector ROR was $13 \mathrm{~cm}$. There is currently only $1 \mathrm{HCB}$ collimator available in our laboratory, so to produce projections for triple-HCB or $\mathrm{HCB}+\mathrm{HCB}+$ fanbeam configurations, multiple single-camera scans were obtained and combined. $\mathrm{CO}$ data were acquired using continuous gantry rotation for $360^{\circ}$, with $1^{\circ}$ sampling. $\mathrm{HCB} \mathrm{CH}$ data were acquired in 2 steps: $300^{\circ}$ revolution of the cameras around the motionless phantom, followed by additional $60^{\circ}$ revolution with continuous phantom translation in the axial direction. During the helical portion of the scan, the phantom traveled $14.2 \mathrm{~cm}$ with approximately constant speed and completely exited the camera FOV by the end of the scan. This procedure was repeated twice to emulate the triple-HCB data, using the single available HCB collimator. The initial angles of the detector equipped with the HCB collimator were $0^{\circ}, 120^{\circ}$, and $240^{\circ}$ for the 3 separate scans. The axial positions of the phantom for each of the scans were identical.

To resemble clinical patient brain scans at Duke, we acquired about 3 million counts per camera equipped with the fanbeam collimator. Throughout the experiment, scan times were adjusted for ${ }^{99 \mathrm{~m}} \mathrm{Tc}$ decay and were equal for all collimator configurations. For instance, if the HCB + HCB + fanbeam scan started immediately after a 15 -min triple-fanbeam scan, the adjusted scan time was then $15.43 \mathrm{~min}$.

All data acquisitions used a $20 \%$ energy window centered at the $140-\mathrm{keV}$ photon emittance peak. The data were acquired on a $256 \times 128$ projection grid. Iterative OSEM reconstruction was used with 6 subsets and 5 iterations. The number of subsets for the experimental studies, compared with the simulation studies, was reduced because of increased noise in the experimental data. As a result, we reduced the noise in the reconstructed images and avoided postfiltering. Photon attenuation was modeled with the "effective" attenuation coefficient $\mu=0.12 \mathrm{~cm}^{-1}$ (20), using a uniform map based on the brain phantom dimensions. Collimator point-spread response and scatter were not modeled in the reconstruction algorithm. Reconstructed image quality was evaluated on the basis of the level of noise, visibility of the lesions, and presence of artifacts in the images.

\section{Patient Imaging}

An interictal brain scan of an adult male patient was performed using the triple-camera SPECT system described earlier. Data acquisition for our research study followed the clinical scan and started $80 \mathrm{~min}$ after the intravenous administration of $10^{9} \mathrm{~Bq}$ (27.5 mCi) of $99 \mathrm{~m} \mathrm{Tc}$-hexamethylpropyleneamine oxime. Our research scan protocol allowed for two 15-min scans with $3 \gamma$-cameras equipped with $1 \mathrm{HCB}$ and 2 fanbeam collimators (collimator specifications are presented in Table 1). During each scan, the cameras rotated continuously for $360^{\circ}$, with $2^{\circ}$ sampling. The data from the second scan were reordered and combined with the data from the first scan to reproduce 2 collimator configurations of interest: $\mathrm{HCB}+\mathrm{HCB}+$ fanbeam and triple-fanbeam. Both configurations contained data from a $15-\mathrm{min} 360^{\circ}$ scan. Thus, we acquired roughly half of the counts typically acquired during a 25-min clinical brain scan. Unavailability of more HCB collimators and a short time frame prevented us from performing a triple$\mathrm{HCB} \mathrm{CH}$ scan for comparison. $\mathrm{HCB}+\mathrm{HCB}+$ fanbeam and triple-fanbeam data were compared for reconstructed image quality and the level of noise in the images.

The camera ROR was $13.5 \mathrm{~cm}$. The photon detection energy window used during data acquisition was 20\% (126-154 keV). The acquisition matrix size was $128 \times 64$. Images were reconstructed iteratively with 6 subsets and 5 iterations. Attenuation was modeled with the coefficient $\mu=0.12 \mathrm{~cm}^{-1}$, using a uniform map based on the dimensions of the head. Scatter was not modeled during image reconstruction.

The Institutional Review Board approved the study, and informed consent was obtained from the patients before the scan.

\section{RESULTS}

\section{Collimator Efficiencies}

Collimator efficiencies, calculated using Monte Carlo simulation of the disk brain phantom, are shown in Table 2. Simulations were performed with and without photon scatter modeling. Similar ratios between efficiencies with and without scatter (Table 2) indicate that scatter is mostly independent of the collimator types and acquisition trajectories studied here. Because scatter does not affect our comparative collimator and acquisition studies, it is excluded from further consideration. Contrary to scatter, photon attenuation depends on the collimator geometry. For the axially converging HCB geometry, photons emitted from the top (cephalic) portions of the cylindric brain phantom are attenuated less than the bottom (caudal) photons because of a shorter attenuation path. This is confirmed by a slice-by-slice triple-camera efficiency plot shown in Figure $3 \mathrm{~A}$ for different collimator combinations and acquisition trajectories. For combinations involving HCB collimators, the photon detection efficiency is increased for the top 2 disks of the simulated disk brain phantom. On the basis of the plot, the $\mathrm{HCB}+\mathrm{HCB}+$ fanbeam combination

\begin{tabular}{|c|c|c|c|}
\hline $\begin{array}{l}\text { Collimator and } \\
\text { acquisition } \\
\text { type }\end{array}$ & $\begin{array}{l}\text { Efficiency } \\
\text { with scatter } \\
\left(\times 10^{-5}\right)\end{array}$ & $\begin{array}{c}\text { Efficiency } \\
\text { without } \\
\text { scatter }\left(\times 10^{-5}\right)\end{array}$ & $\begin{array}{l}\text { Efficiency with } \\
\text { scatter/efficiency } \\
\text { without scatter }\end{array}$ \\
\hline $\mathrm{HCB}, \mathrm{CO}$ & $7.39 \pm 0.10$ & $5.63 \pm 0.08$ & $1.31 \pm 0.03$ \\
\hline $\mathrm{HCB}, \mathrm{CH}$ & $6.70 \pm 0.12$ & $5.10 \pm 0.09$ & $1.31 \pm 0.03$ \\
\hline $\mathrm{FB}, \mathrm{CO}$ & $5.26 \pm 0.07$ & $4.05 \pm 0.06$ & $1.30 \pm 0.03$ \\
\hline $\mathrm{FCB}, \mathrm{CO}$ & $4.98 \pm 0.11$ & $3.90 \pm 0.09$ & $1.28 \pm 0.04$ \\
\hline $\mathrm{PB}, \mathrm{CO}$ & $2.94 \pm 0.07$ & $2.24 \pm 0.05$ & $1.31 \pm 0.04$ \\
\hline \multicolumn{4}{|c|}{$\mathrm{FB}=$ fanbeam. } \\
\hline
\end{tabular}



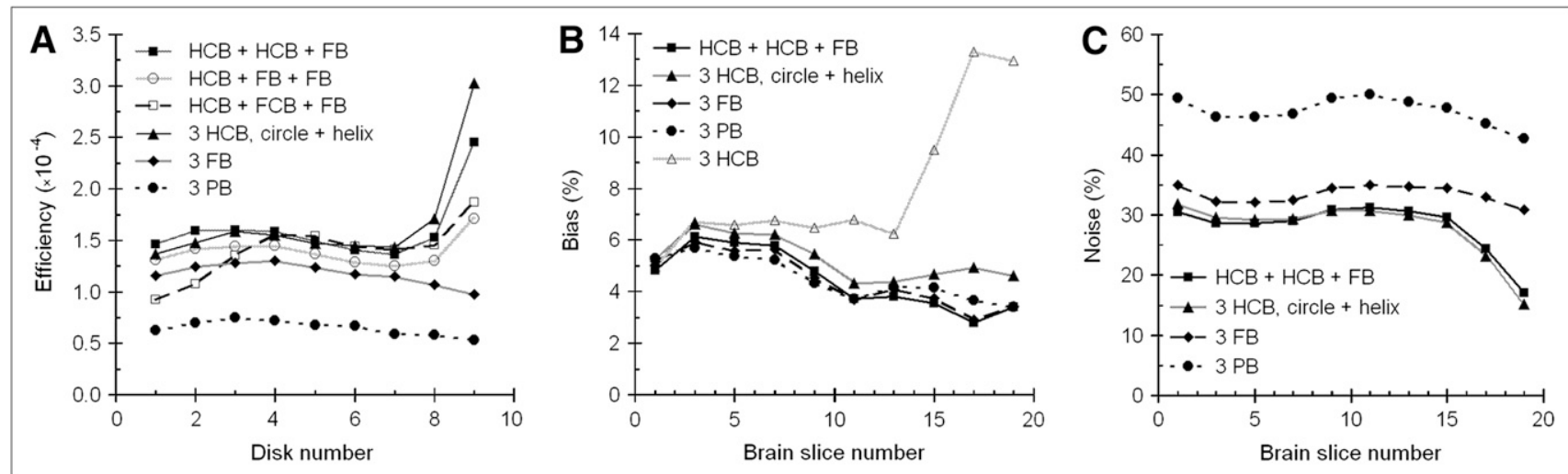

FIGURE 3. Presented for different collimator combinations and acquisition trajectories are slice-by-slice photon detection efficiencies calculated using Monte Carlo simulation (A), reconstruction bias plot for the Hoffman 3D brain phantom simulated using ray-driven method (B), and levels of noise in reconstructed images of simulated Hoffman brain phantom (C). Acquisition trajectories included a $\mathrm{CO}$ (not specified in legend) and $\mathrm{CH}$ trajectory (specified in legend). Disks and slices are numbered in caudal-to-cranial order.

with $\mathrm{CO}$ acquisition and the triple-HCB collimation with a $\mathrm{CH}$ acquisition trajectory offer the highest efficiencies for every part of the disk brain phantom. The combination that includes the FCB collimator reduces the efficiency at the base of the phantom because of truncation and will not be discussed further in this article. The HCB + fanbeam + fanbeam combination is less efficient than the $\mathrm{HCB}+$ $\mathrm{HCB}+$ fanbeam combination and will also be omitted from further consideration. Triple-fanbeam and triple-PB collimations will be considered as conventional references.

\section{Reconstructed Image Quality: Bias, Noise, and SNR}

Hoffman 3D brain phantom SPECT was simulated for a triple-camera system using ray-driven forward-projection and iterative OSEM reconstruction algorithms. Noise-free reconstructions were compared with Hoffman brain phantom bitmaps to estimate reconstruction bias. Large bias values may indicate that axial sampling artifacts are present in the reconstructed images. The bias is shown in Figure 3B for all 19 slices of the phantom. Triple-HCB CO acquisition was simulated to demonstrate the extent of artifacts due to insufficient axial sampling in terms of bias values that gradually increase toward the top of the brain. The plot demonstrates that both adding a helical component to the triple-HCB acquisition trajectory and combining $2 \mathrm{HCB}$ collimators with fanbeam markedly improve axial sampling of the phantom. The bias values of the $\mathrm{HCB}+\mathrm{HCB}+$ fanbeam combination are comparable to triple-fanbeam bias, confirming quantitatively that reconstructed images do not display any axial artifacts. Triple-HCB $\mathrm{CH}$ bias values are slightly larger, especially toward the top of the brain. This is primarily due to the limited helical component of the scan. Increasing the number of helical views reduces the bias but decreases the photon detection efficiency. Nevertheless, visual analysis of the reconstructed images in our study suggests that the artifacts are hardly noticeable and should be tolerable for practical (clinical) purposes.
Along with noise-free reconstructions, we generated an ensemble of 32 noisy projection data samples for simulation studies. The noise level in the reconstructed images was evaluated slice by slice using Equation 2. The averaged results are shown in Figure 3C for all 19 slices of the simulated phantom. As expected, both the $\mathrm{HCB}+\mathrm{HCB}+$ fanbeam combination and the triple- $\mathrm{HCB} \mathrm{CH}$ acquisition offer reduced noise in the reconstructed images due to increased photon detection efficiency, particularly at the top of the brain. Slight efficiency advantage of the HCB + $\mathrm{HCB}+$ fanbeam combination at the bottom of the brain is expected and is also visible in the plot (Fig. 3C).

Reconstructed images of the simulated Hoffman brain phantom are shown in Figure 4 for all 4 acquisition types. Brain slices containing lesions are displayed. Qualitative analysis of the brain images shows reduced noise due to increased sensitivity at the same spatial resolution and confirms the advantages of the $\mathrm{HCB}+\mathrm{HCB}+$ fanbeam combination and triple- $\mathrm{HCB} \mathrm{CH}$ acquisition over triplefanbeam and triple-PB CO scans. No artifacts were detected.

Quantitatively, performances of the studied configurations were evaluated using SNRs of the lesions. Table 3 shows the SNR values for 6 lesions in the brain phantom, averaged over an ensemble of 32 noise realizations. Higher magnitudes of the SNR for the HCB + HCB + fanbeam combination and triple-HCB $\mathrm{CH}$ acquisition is evidence of noise reduction and better lesion visualization because of increased sensitivity. The uncertainties in the average SNR values $\left(\delta_{<\text {SNR }}\right)$ ) in Table 3 were calculated as follows:

$$
\delta_{<\mathrm{SNR}>}=\frac{\sigma_{\mathrm{SNR}}}{\sqrt{\mathrm{N}}}
$$

Eq. 4

where

$$
\sigma_{\mathrm{SNR}}=\sqrt{\frac{1}{\mathrm{~N}-1} \sum_{\mathrm{i}=1}^{\mathrm{N}}\left(\mathrm{SNR}_{\mathrm{i}}-<\mathrm{SNR}>\right)^{2}} \quad \text { Eq. } 5
$$


A

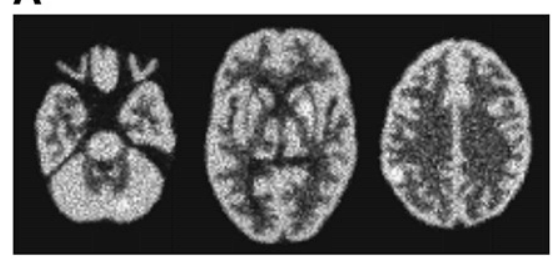

C

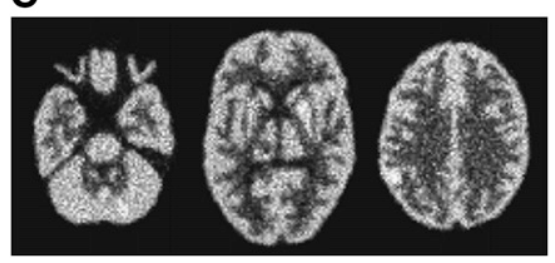

B

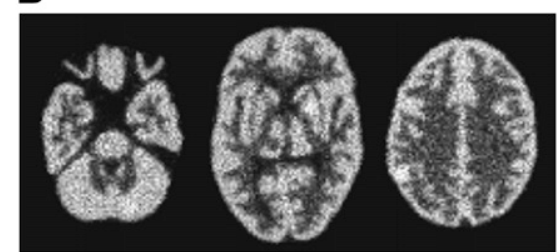

D

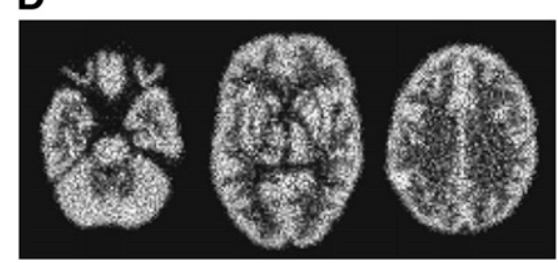

FIGURE 4. Simulated Hoffman brain phantom scan results for 4 acquisition types: HCB + HCB + fanbeam CO (A), triple-HCB $\mathrm{CH}$ trajectory (B), triplefanbeam (C), and triple-PB COs (D). Reconstructed images of brain phantom slices containing hot and cold lesions are shown. is the SD of the SNR distribution for $\mathrm{N}=32$ noise realizations, assuming gaussian distribution of the SNR values. The errors can be reduced using a larger number of noise realizations.

\section{Experimental Brain Phantom Imaging}

The physical Hoffman brain phantom was scanned using the triple-camera SPECT system and 4 acquisition types described earlier. Acquisition times for each type, corrected for ${ }^{99 \mathrm{~m}} \mathrm{Tc}$ decay, were the same. The number of photons detected during each acquisition scenario is shown in Table 4. There was no truncation throughout the scan.

Reconstructed images of the Hoffman brain phantom are shown in Figure 5 for all 4 acquisition types. Brain slices containing cold lesions in the cerebellum, thalamus, and frontal lobe are displayed. The noise is reduced with the $\mathrm{HCB}+\mathrm{HCB}+$ fanbeam collimator combination and triple-HCB $\mathrm{CH}$ acquisition types, compared with triplefanbeam and triple-PB CO scans. No apparent axial sampling artifacts were observed.

\section{Patient Imaging Data}

Two 15-min interictal brain scans were obtained for a patient using the triple-camera SPECT system described

\begin{tabular}{|c|c|c|c|c|}
\hline & \multicolumn{4}{|c|}{ Acquisition type } \\
\hline \multirow[b]{2}{*}{ Lesion* } & \multicolumn{4}{|l|}{$\mathrm{HCB}+\mathrm{HCB}$} \\
\hline & $+F B$ & $3 \mathrm{HCB}, \mathrm{CH}$ & $3 \mathrm{FB}, \mathrm{CO}$ & $3 \mathrm{~PB}, \mathrm{CO}$ \\
\hline Cold 1 & $-2.55 \pm 0.06$ & $-2.41 \pm 0.05$ & $-2.17 \pm 0.05$ & $-1.43 \pm 0.04$ \\
\hline Hot 1 & $1.39 \pm 0.04$ & $1.30 \pm 0.04$ & $1.26 \pm 0.04$ & $0.86 \pm 0.05$ \\
\hline Cold 2 & $-2.10 \pm 0.04$ & $-1.99 \pm 0.04$ & $-1.77 \pm 0.04$ & $-1.13 \pm 0.05$ \\
\hline Hot 2 & $1.45 \pm 0.05$ & $1.57 \pm 0.05$ & $1.29 \pm 0.05$ & $0.90 \pm 0.05$ \\
\hline Cold 3 & $-2.64 \pm 0.06$ & $-2.75 \pm 0.06$ & $-2.33 \pm 0.05$ & $-1.45 \pm 0.04$ \\
\hline Hot 3 & $3.68 \pm 0.07$ & $3.61 \pm 0.05$ & $3.17 \pm 0.07$ & $2.27 \pm 0.05$ \\
\hline 1 & & al-to-cranial & & \\
\hline
\end{tabular}

earlier. We acquired $4.36 \times 10^{6}$ photons with the $\mathrm{HCB}+$ $\mathrm{HCB}+$ fanbeam collimator combination and $2.99 \times 10^{6}$ photons with a triple-fanbeam CO scan, which is half of the data acquired during a longer clinical interictal scan of the same patient. Scan times were equal in both cases. Reconstructed patient brain images are shown in Figures 6A and 6B. The images demonstrate improved quality with the $\mathrm{HCB}+\mathrm{HCB}+$ fanbeam combination due to increased efficiency and reduced noise. To further reduce noise, images can be postfiltered using the Hann filter, with a cutoff frequency of 1.4 cycles/cm (Figs. 6C and 6D).

\section{DISCUSSION}

Statistical noise in SPECT reconstructions directly affects the image quality. The use of converging HCB collimatorsdesigned specifically for brain SPECT-compared with conventional parallel-beam and fanbeam collimators used in clinical studies, improves sensitivity and reduces noise in images at the same or better resolution. To avoid axial sampling artifacts with HCB collimation typically occurring during $\mathrm{CO}$ acquisition, $\mathrm{HCB}$ can be combined with a fanbeam collimator in multicamera SPECT systems. Alternatively, helical HCB acquisition has been previously proven to markedly improve axial sampling. In this article, we studied various collimator combinations and acquisition types for a triple-camera SPECT system. The objective of this study was to find a combination that would offer

\begin{tabular}{|c|c|}
\hline Acquisition type & No. of photons $\left(\times 10^{6}\right)$ \\
\hline $\mathrm{HCB}+\mathrm{HCB}+\mathrm{FB}, \mathrm{CO}$ & 13.55 \\
\hline $3 \mathrm{HCB}, \mathrm{CH}$ & 14.39 \\
\hline $3 \mathrm{FB}, \mathrm{CO}$ & 9.31 \\
\hline $3 \mathrm{~PB}, \mathrm{CO}$ & 6.39 \\
\hline $\mathrm{FB}=$ fanbeam. & \\
\hline
\end{tabular}


A

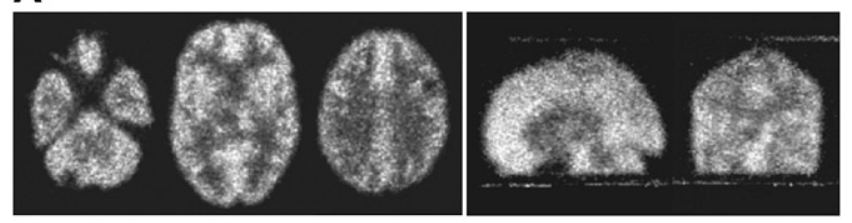

B

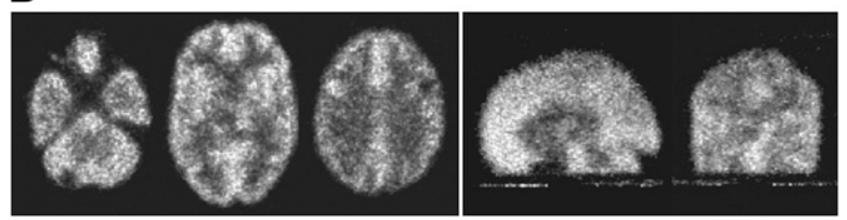

C

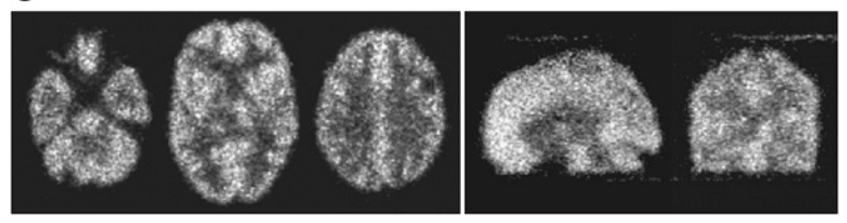

D

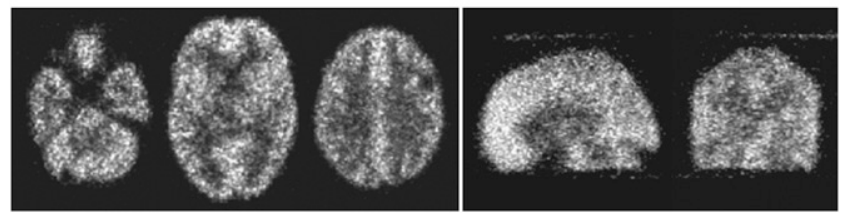

FIGURE 5. Experimental Hoffman brain phantom scan results for 4 acquisition types: $\mathrm{HCB}+\mathrm{HCB}+$ fanbeam $\mathrm{CO}$ $(\mathrm{A})$, triple-HCB $\mathrm{CH}$ trajectory $(\mathrm{B})$, triple-fanbeam $(\mathrm{C})$, and triple-PB COs (D). Transaxial views of reconstructed brain phantom slices containing 3 cold lesions are shown, along with centrally located sagittal and coronal slices of phantom. Each slice is summed with 2 adjacent slices, to reduce noise.

both increased sensitivity and artifact-free image reconstruction.

The Monte Carlo simulation study emphasized 2 acquisition types that offer the highest sensitivity: $\mathrm{HCB}+\mathrm{HCB}+$ fanbeam collimator combination with a $\mathrm{CO}$ scan and
triple-HCB $\mathrm{CH}$ acquisition. Both acquisition types offered $2 \times 10^{-4}$ photon detection efficiency. Given that $\mathrm{HCB}$ collimation, compared with fanbeam, offers $40 \%$ more detected photons and fanbeam provides sufficient axial sampling, the advantage of their combination is logical. The $\mathrm{HCB}+\mathrm{HCB}+$ fanbeam collimator combination studied here was $27 \%$ more efficient than triple-fanbeam (efficiency, $1.58 \times 10^{-4}$ ) and 2.27 times more efficient than the triple-parallel-hole collimation (efficiency, $0.88 \times$ $10^{-4}$ ), with equal spatial resolution measured on the axis of rotation. The advantage is even greater for converging collimators with shorter focal lengths, provided the detector FOV is large enough to avoid truncation. Other collimator combinations were also studied but were less efficient than the HCB + HCB + fanbeam configuration. To be comparable in terms of sensitivity, the triple- $\mathrm{HCB} \mathrm{CH}$ acquisition had to have a much longer circular component than helical: 300 circular views to 60 helical views in our study. The limited number of axial (helical) views caused insufficient axial sampling, but the artifacts were hardly visible in noise-free reconstructions and not visible in noisy images. Reconstruction bias analysis confirmed that the addition of a fanbeam collimator to 2 HCBs markedly improves axial sampling and eliminates artifacts otherwise present during the HCB CO acquisition. In both simulation and experiment, the reconstructed Hoffman brain phantom images demonstrated higher quality (Figs. 4 and 5) and better lesion visualization (SNR values, Table 3) for the HCB + $\mathrm{HCB}+$ fanbeam combination and triple- $\mathrm{HCB} \mathrm{CH}$ acquisition than did the triple-fanbeam and triple-PB CO scans. The SNR values for the HCB + HCB + fanbeam combination were on average $15 \%$ higher-corresponding to a $1 \mathrm{SD}\left(\sigma_{\mathrm{SNR}}\right)$ shift of the SNR distribution-than were the triple-fanbeam collimation values and $72 \%$ higher than were the triple-PB acquisitions, which is almost $4 \sigma_{\mathrm{SNR}}$ difference. To see if the differences between these samples are statistically significant, we performed a standard 2 -sample $z$ test of the statistical hypothesis. Under the null hypothesis, which assumes that there is no difference between the triple-fanbeam (or parallel) collimation and the $\mathrm{HCB}+\mathrm{HCB}+$ fanbeam combination, the distance
A

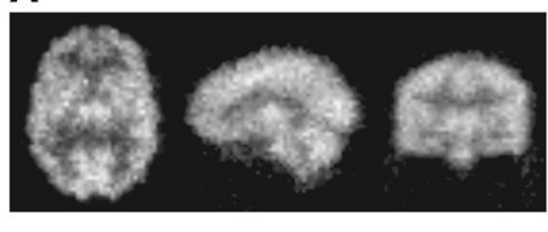

C fanbeam $\mathrm{CO}$ acquisitions (B). Threeslice summing is used to reduce noise in $A$ and $B$. Postfiltered images are shown for $\mathrm{HCB}+\mathrm{HCB}+$ fanbeam $(\mathrm{C})$ and triple-fanbeam (D) acquisition types.
B

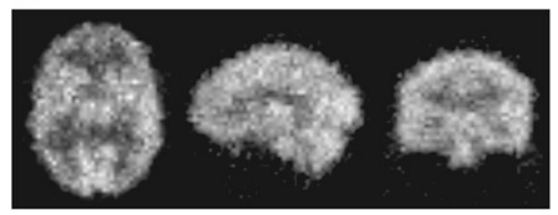

D

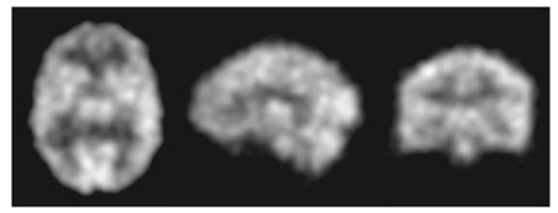


between 2 distribution means in units of the SE of the SNR mean is calculated as follows:

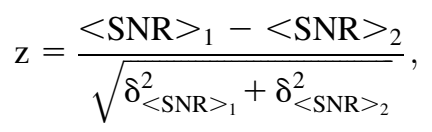

where indices 1 and 2 correspond to the $\mathrm{HCB}+\mathrm{HCB}+$ fanbeam combination and the triple-fanbeam (or parallel) collimations. On the basis of the data in Table 3, the average value of $z$ is 4 for triple-fanbeam and 13 for triple-parallel SNR distributions. Both $z$ scores are large enough $(P>$ $0.9999)$ to conclude that our null hypothesis can be rejected, establishing that the differences between the means of the SNR distributions for different collimator combinations are statistically significant with more than $95 \%$ confidence level.

Patient brain scans confirmed the pattern: $46 \%$ more photons, albeit with slightly lower resolution (collimator resolutions, Table 1), were detected with the HCB + HCB + fanbeam combination, compared with a triple-fanbeam scan. This resulted in noise reduction and improved image quality (Fig. 6). Hence, the $\mathrm{HCB}+\mathrm{HCB}+$ fanbeam combination can be used to either improve image quality or reduce the patient scan time by $30 \%$.

The study can also be applied to dual-camera SPECT systems, because triple-camera systems are not currently mass-produced in the United States. After the results, it is reasonable to assume that the $\mathrm{HCB}+$ fanbeam combination and dual-HCB $\mathrm{CH}$ acquisitions will perform better than dual-fanbeam or dual-PB acquisitions. In our case, the efficiency advantage of the HCB + fanbeam combination over fanbeam + fanbeam would be $20 \%$. For single-camera systems, combining collimators is not possible, and using $\mathrm{CH}$ acquisition may result in greater axial distortions due to insufficient axial sampling. However, multiple scans may be performed with a single camera to emulate dual- or triple-camera SPECT. The popularity of triple-camera systems may depend on the popularity of brain SPECT. Recent development and advancement of new SPECT radiopharmaceuticals targeted for specific neurologic disorders (e.g., Parkinson disease) $(21,22)$ may increase triple-camera applications in brain imaging.

From a technical point of view, combining HCB and fanbeam collimators for a $\mathrm{CO}$ scan is easier than implementing $\mathrm{HCB} \mathrm{CH}$ acquisition, which requires careful measurements of the patient translation in the axial direction. For some systems, the bed tilt, direction shift, and variable speed during translation can be significant and may need to be considered during reconstructions.

\section{CONCLUSION}

We demonstrated that a triple-camera SPECT system equipped with a combination of $2 \mathrm{HCB}$ and 1 fanbeam collimators, compared with conventional parallel-hole and fanbeam geometries, offers improved brain imaging by increasing sensitivity and reducing statistical noise at equal spatial resolution. This combination also eliminates axial sampling artifacts typically observed in cone-beam $\mathrm{CO}$ scans due to insufficient axial sampling. Both qualitative and quantitative analyses of the reconstructed images in simulation, experimental brain phantom, and patient brain scans confirm that the HCB + HCB + fanbeam combination outperforms other acquisition types and offers improved image quality due to reduced noise. Triple-HCB CH acquisition has shown competitive results but is slightly harder to implement and may result in minor and tolerable axial sampling artifacts due to a limited number of axial (helical) views. The combination of $2 \mathrm{HCB}$ and 1 fanbeam collimators in a triple-camera SPECT system has the potential for high-sensitivity brain imaging.

\section{ACKNOWLEDGMENTS}

This work is supported by the National Institute of Neurological Disorders and Stroke of the National Institutes of Health (NIH) under grant R01 NS054797. Experimental and patient scan data were acquired using shared instrumentation supported by the NIH National Center for Research Resources under grant S10 RR15697.

\section{REFERENCES}

1. Li J, Jaszczak RJ, Van Mullekom A, Scarfone C, Greer KL, Coleman RE. Halfcone beam collimation for triple-camera SPECT systems. J Nucl Med. 1996;37: 498-502.

2. Ter-Antonyan R, Jaszczak RJ, Bowsher JE, Greer KL, Metzler SD. Brain SPECT simulation using half-cone-beam collimation and single-revolution helical-path acquisition. IEEE Trans Nucl Sci. 2007;54:475-479.

3. Ter-Antonyan R, Jaszczak RJ, Bowsher JE, Greer KL, Metzler SD. Quantitative evaluation of half-cone-beam scan paths in triple-camera brain SPECT. IEEE Trans Nucl Sci. 2008;55:2518-2526.

4. Danielsson P-E, Edholm P, Magnusson-Seger M. Towards exact 3D-reconstruction for helical cone-beam scanning of long objects: a new detector arrangement and a new completeness condition. Paper presented at: International Meeting on Fully Three-Dimensional Image Reconstruction in Radiology and Nuclear Medicine; June 25-27, 1997; Pittsburgh, PA.

5. Zeng GL, Gullberg GT. Helical SPECT using axially truncated data. IEEE Trans Nucl Sci. 1999;46:2111-2118.

6. Kudo H, Noo F, Defrise M. Cone-beam filtered-backprojection algorithm for truncated helical data. Phys Med Biol. 1998;43:2885-2909.

7. Metzler SD, Greer KL, Jaszczak RJ. Helical pinhole SPECT for small-animal imaging: a method for addressing sampling completeness. IEEE Trans Nucl Sci. 2003;50:1575-1583.

8. Kalender WA. Principles and performance of spiral CT. In: Goldman LW, Fowlkes JB, eds. Medical CT and Ultrasound: Current Technology and Applications. Madison, WI: Advanced Medical Publishing; 1995:379-410.

9. Tam KC, Samarasekera S, Sauer F. Exact cone-beam CT with a spiral scan. Phys Med Biol. 1998;43:1015-1024.

10. Schaller S, Noo F, Sauer F, Tam KC, Lauritsch G, Flohr T. Exact radon rebinning algorithm for the long object problem in helical cone-beam CT. IEEE Trans Med Imaging. 2000;19:361-375.

11. Jaszczak RJ, Li J, Wang J, Coleman RE. Three-dimensional SPECT reconstruction of combined cone beam and parallel beam data. Phys Med Biol. 1992;37:535-548.

12. Li J, Jaszczak RJ, Turkington TG, Greer KL, Coleman RE. SPECT reconstruction of combined cone beam and parallel hole collimation with experimental data. IEEE Trans Nucl Sci. 1993;40:300-306.

13. Gullberg GT, Zeng GL. Cardiac single-photon emission-computed tomography using combined cone-beam/fan-beam collimation. IEEE Trans Nucl Sci. 2005; 52:143-153.

14. Stone CD, Smith MF, Greer KL, Jaszczak RJ. A combined half-cone beam and parallel hole collimation system for SPECT brain imaging. IEEE Trans Nucl Sci. 1998;45:1219-1224. 
15. Defrise M, Clack R. Filtered backprojection reconstruction of combined parallel beam and cone beam SPECT data. Phys Med Biol. 1995;40:15171537.

16. Park M-A, Moore SC, Kijewski MF. Brain SPECT with short focal-length conebeam collimation. Med Phys. 2005;32:2236-2244.

17. Lalush DS, DiMeo AJ. An observer study evaluating dual-plane circular-orbit cone-beam brain SPECT. J Nucl Med. 2002;43:1578-1583.

18. Ter-Antonyan R, Jaszczak RJ, Bowsher JE, Greer KL, Metzler SD. Triplecamera brain SPECT combining convergent collimators [abstract]. J Nucl Med. 2008;49(suppl):124P
19. Hudson HM, Larkin RS. Accelerated image reconstruction using ordered subsets of projection data. IEEE Trans Med Imaging. 1994;13:601-609.

20. Jaszczak RJ, Greer KL, Floyd CE, Harris CC, Coleman RE. Improved SPECT quantification using compensation for scattered photons. J Nucl Med. 1984;25: 893-900.

21. Tzen K-Y, Lu C-S, Yen T-C, Wey S-P, Ting G. Differential diagnosis of Parkinson's disease and vascular parkinsonism by $99 \mathrm{~m}$ Tc-TRODAT-1. J Nucl Med. 2001;42:408-413.

22. Lu C-S, Weng YH, Chen MC, et al. ${ }^{99 \mathrm{~m} T c-T R O D A T-1}$ imaging of multiple system atrophy. J Nucl Med. 2004;45:49-55. 